\title{
Advanced HIV Disease Management Practices within Inpatient Medicine Units at a Referral Hospital in Zambia: A Retrospective Chart Review
}

Nyuma Mbewe ( $\square$ nymbewe@gmail.com )

University of Zambia School of Medicine https://orcid.org/0000-0002-3309-0538

Michael J. Vinikoor

University Teaching Hospital

Sombo Fwoloshi

University Teaching Hospital

Mundia Mwitumwa

University Teaching Hospital

Shabir Lakhi

University Teaching Hospital

Suilanji Sivile

University Teaching Hospital

Mallika Yavatkar

University of Maryland School of Medicine

Brianna Lindsay

University of Maryland School of Medicine

Kristen Stafford

University of Maryland School of Medicine,

Lottie Hachaambwa

University Teaching Hospital

Lloyd Mulenga

University Teaching Hospital

Cassidy W. Claassen

University Teaching Hospital

Short report

Keywords: Advanced HIV Disease, TB diagnostics, Viral Load Monitoring, sub-Saharan Africa

Posted Date: November 8th, 2021

DOI: https://doi.org/10.21203/rs.3.rs-1042337/v1 
License: (c) (i) This work is licensed under a Creative Commons Attribution 4.0 International License. Read Full License

Version of Record: A version of this preprint was published at AIDS Research and Therapy on February 22nd, 2022. See the published version at https://doi.org/10.1186/s12981-022-00433-8. 
Advanced HIV disease management practices within inpatient medicine units at a referral hospital in Zambia: a retrospective chart review

Nyuma Mbewe ${ }^{1}$; Michael J. Vinikoor, ${ }^{1,2}$; Sombo Fwoloshi' ${ }^{1}$; Mundia Mwitumwa ${ }^{1}$; Shabir Lakhi ${ }^{1}$; Suilanji Sivile ${ }^{1}$; Mallika Yavatkar ${ }^{3}$, Brianna Lindsay ${ }^{4,5}$; Kristen Stafford ${ }^{4,5}$; Lottie Hachaambwa ${ }^{1,4,5}$; Lloyd Mulenga ${ }^{1,6,7,8}$; Cassidy W. Claassen ${ }^{1,4,5}$

${ }^{1}$ Adult Infectious Diseases Center, University Teaching Hospital, Lusaka, Zambia

${ }^{2}$ University of Alabama at Birmingham School of Medicine, Birmingham, Alabama, USA

${ }^{3}$ University of Maryland School of Medicine, Baltimore, Maryland, USA

${ }^{4}$ Institute of Human Virology, Division of Infectious Diseases, University of Maryland School of Medicine, Baltimore, Maryland, USA

${ }^{5}$ Center for International Health, Education, and Biosecurity, University of Maryland School of Medicine, Baltimore, Maryland, USA

${ }^{6}$ Vanderbilt University Medical Center (VUMC), Department of Medicine, Division of Infectious Diseases, Nashville, Tennessee, USA

${ }^{7}$ Vanderbilt Institute for Global Health (VIGH), Nashville, Tennessee, USA

${ }^{8}$ Ministry of Health, Ndeke House, Lusaka, Zambia

Key words: Advanced HIV Disease, TB diagnostics, Viral Load Monitoring, sub-Saharan Africa

\section{Corresponding Author:}

Nyuma Mbewe (nymbewe@gmail.com) 


\section{ABSTRACT}

\section{Background}

Zambia recently achieved UNAIDS 90-90-90 treatment targets for HIV epidemic control; however, inpatient facilities continue to face a large burden of patients with advanced HIV disease and HIV-related mortality. Management of advanced HIV disease, following guidelines from outpatient settings, may be more difficult within complex inpatient settings. We evaluated adherence to HIV guidelines during hospitalization, including opportunistic infection (OI) screening, treatment, and prophylaxis.

\section{Methods}

We reviewed inpatient medical records of people living with HIV (PLHIV) admitted to the University Teaching Hospital in Lusaka, Zambia between December 1, 20218 and April 30, 2019. We collected data on patient demographics, antiretroviral therapy (ART), HIV biomarkers, and OI screening and treatment - including tuberculosis (TB), Cryptococcus, and OI prophylaxis with cotrimoxazole (CTX). Screening and treatment cascades were constructed based on the 2017 WHO Advanced HIV Guidelines.

\section{Results}

We reviewed files from 200 charts of patients with advanced HIV disease; of these $92 \%$ (184/200) had been on ART previously; $58.1 \%$ (107/184) for more than 12 months. HIV viral load (VL) testing was uncommon but half of VL results were high. 39\% (77/200) of patients had a documented CD4 count result. Of the 172 patients not on anti-TB treatment (ATT) on admission, TB diagnostic tests (either sputum Xpert MTB/RIF MTB/RIF or urine TB-LAM) were requested for $105(61 \%)$ and resulted for 60 of the 105 (57\%). Nine of the 14 patients (64\%) with a positive lab result for TB died before results were available. Testing for Cryptococcosis was performed predominantly in patients with symptoms of meningitis. Urine TB-LAM testing was rarely performed.

\section{Conclusions}

Inconsistent CD4 testing reduced recognition of advanced HIV and OI screening was suboptimal, in part due to laboratory challenges. HIV programs can potentially reduce mortality and identify PLHIV with retention and adherence issues through strengthening inpatient activities, including VL testing.

\section{Word count: 298}




\section{Abbreviations:}

AHD - Advanced HIV disease

ART - Antiretroviral therapy

ATT - Antituberculosis therapy

$\mathrm{CrAg}$ - Cryptococcal Antigen Test

CSF - Cerebral Spinal Fluid

CTX - Co-trimoxazole

HIV - Human Immunodeficiency Virus

IQR - Interquartile range

LIS - Laboratory Information System

LMIC - Lower - Middle Income countries

Ols - Opportunistic Infections

PLHIV - People living with HIV

SD - Standard Deviation

TB- Tuberculosis

TB- LAM - lateral flow urine lipoaribomannan assay for tuberculosis

TPT - TB Preventative Therapy

$\mathrm{VL}-$ Viral Load test

UTH - University Teaching Hospital

WHO - World Health Organization

ZamPHIA - Zambia Population -based HIV Impact Assessment 


\section{Introduction}

Global scale up of antiretroviral therapy (ART) has attained high coverage levels and prevented over 12.1 million HIV-related deaths worldwide, yet many clients still present with advanced HIV disease. (1-3). Declines in HIV-related mortality and morbidity observed between 2003 to 2015 have plateaued, particularly in sub-Saharan Africa(4) In Zambia, mortality among people living with HIV (PLHIV) following linkage to care remains high, at 10.3 per 100 personyears among men with at least 18 months of ART (compared to 5.5 per 100 person years among women) which is slightly higher than the 6.7 and 6.9 per 100 person years reported in South Africa and Ethiopia respectively(5-7). Additionally, post discharge mortality among PLHIV was found to be $22 \%$ at the largest hospital in Zambia(3). While there is growing interest in noncommunicable diseases among PLHIV (8), it is likely that HIV-associated mortality is partly driven by the prevalence of advanced HIV disease, defined as HIV infection with CD4 count $<200$ cells $/ \mathrm{mm}^{3}$ or WHO clinical stage 3 or 4 disease (9). A recent study from the Botswana HIV treatment and care program revealed that $24 \%$ of patients who initiated ART in the 'Test and Start' era had advanced disease (10).

Guidelines were developed by the World Health Organization (WHO) for the detection and management of advanced HIV in 2017, based on randomized controlled trials and expert opinion (9,11). These guidelines emphasize the need for CD4 count testing and diagnosis, treatment, and prevention of opportunistic infections (OIs) such as tuberculosis and cryptococcosis, the Ols that are responsible for a large proportion of HIV-related morbidity and mortality (9). 
Almost two decades into the widespread availability of ART in Lower-Middle Income Countries (LMIC), most PLHIV are treatment experienced, and the guidelines have expanded to include ART monitoring (9). However, much of the data and expert opinion underpinning these guidelines were derived in outpatient models of chronic care and several landmark clinical trials that enrolled ART-naïve outpatients, the majority of whom had asymptomatic HIV (12-15). Compared to outpatient and community programs, inpatient settings are more focused on immediate life-saving interventions. This difference could create missed opportunities to recognize and address advanced disease among PLHIV.

We evaluated the implementation of WHO advanced HIV disease care guidelines in the University Teaching Hospital (UTH) in Lusaka, Zambia, a setting with high inpatient HIV prevalence. We sought to identify gaps in requesting and providing recommended tests and treatment and explored timeliness of screening tests at UTH. The purpose of our study was to describe current practices and identify opportunities to reduce the impact of advanced disease on HIV-related mortality.

\section{Methods}

\section{Study Setting}

The UTH is a 1,665-bed capacity hospital and the largest referral center in the country. The six inpatient Internal Medicine wards - collectively known as E-block - are reserved for patients cared for by various Internal Medicine subspecialties. HIV rapid testing is opt-out among all admitted patients who report being HIV negative or unknown status. Requests for laboratory tests other than HIV rapid testing are documented by clinicians in the patient file. Samples are 
collected on the wards and taken to the central laboratories, where test results are entered into a laboratory information system (LIS). The urine lipoaribomannan assay for tuberculosis (TBLAM), which can be performed at the bedside, is documented in the paper file. Once LIS reports results, they are printed for filing in the patient chart. All prescribed medications are listed in a paper tool that accompanies the chart. We performed a retrospective chart review of services provided between $1^{\text {st }}$ December 2018 and 30 ${ }^{\text {th }}$ April 2019 using inpatient records at the end of hospitalization (discharge or death).

\section{Study Procedures}

We identified eligible patients using ward registers, which are the most comprehensive tool available to track E-block admissions. Inclusion criteria was age 16 years or older, HIV infection (based on documentation of self-reported HIV positive or a positive test in the file), and admission to the medical wards under the care of the Infectious Diseases Unit. Patients who left the hospital against medical advice, were excluded from the study (Figure 1). Patients were also excluded if files were inaccessible post discharge; this most often occurred because they opted for post discharge review at their local clinic and not at the UTH. Using a standardized tool, we extracted patient demographic data, presenting symptoms, laboratory testing including HIV biomarkers (CD4 count and VL) and OI screening using the LIS, and pharmacy records related to Ol treatment and prevention.

Our assessment focused on four domains adapted from the 2017 WHO Advanced HIV guidelines(9). First, we assessed ART monitoring as guidelines recommend VL testing in treatment-experienced patients from six months post initiation, then annually if the patient is virologically suppressed and deemed stable. CD4 testing is to be done at baseline then every six 
months subsequently (9). Second, we assessed the proportion of those prescribed OI prophylaxis, as patients with CD4 counts less than 350 cells $/ \mathrm{mm}^{3}$ should be commenced on cotrimoxazole (CTX) prophylaxis. Third, we evaluated TB care. Per guidelines, all patients should be assessed for tuberculosis via the WHO TB screening questions, a urine TB- LAM and/or Gene Xpert MTB/RIF testing. Those who test negative for TB are to receive six months of isoniazid as TB preventative therapy (TPT). Finally, we assessed Cryptococcosis management. For CD4 counts less than 100 cells $/ \mathrm{mm}^{3}$ or with symptoms such as headache and fever suggestive of cryptococcal meningitis, serum Cryptococcal antigen $(\mathrm{CrAg})$ test is warranted and fluconazole prophylaxis until the CD4 count is greater than 350 cells $/ \mathrm{mm}^{3}$.

\section{Statistical Analysis}

Microsoft Excel version 16 (Microsoft Corporation, Seattle, USA) was used to store raw data and R version 4.0.3 (R Core Team, 2021) was used for analyses. We described patient characteristics and pre-hospital use of ART and anti-tuberculous therapy (ATT) on admission to the hospital. Then, we constructed four 'care cascades' as follows: (a) ART monitoring with HIV VL, (b) appropriate use of CTX based on CD4 count $<350$ cells $/ \mathrm{mm}^{3}$, (c) TB testing and treatment, and (d) cryptococcosis diagnostic testing. These cascades were guided by the 2017 WHO Advanced HIV disease guidelines (9), which were incorporated in the Zambia Consolidated Treatment Guidelines as early as 2018(17). When possible, we also described the timing of test resulting in relation to the admission course. Descriptive statistics were used to analyze baseline characteristics. Continuous variables were expressed as median and interquartile range (IQR) 
whilst categorical variables were expressed as a percentage from the group which they were derived.

\section{Ethical Approvals}

The study was approved by UTH, the University of Zambia Biomedical Research Ethics Committee (Ref. 012-02-19), the University of Maryland Baltimore institutional review board (IRB), and the Zambia National Health Research Authority. Given the retrospective nature of the study, a waiver of informed consent was obtained from the IRBs and no patient identifiers were collected.

\section{Results}

\section{$\underline{\text { Baseline Characteristics }}$}

Between $1^{\text {st }}$ December 2018 to $1^{\text {st }}$ May 2019, there were 331 individuals eligible for chart review and 200 were enrolled in the study. We excluded 11 who left against medical advice and 120 who were discharged but did not leave their chart behind against hospital policy. Patient characteristics are reported in Table 1. Among included participants, the average length of stay was 11 days, with a minimum of 1 day and a max of 56 days. A total of 109 (54\%) of participants were female. Median age was 39 years with $76 \%$ below 50 years of age [IQR 31-45yrs]. CD4 count results were frequently unavailable ( $57 \%$ missing), but of those with available data, $62 \%$ of patients had advanced HIV disease (48 patients of the 77 patients with available CD4 results). There was no difference in the percent of patients with documented CD4 cell count among the patients who died compared to those who were discharged ( $28 \%$ and $22 \%$ respectively). 
Table 1: Demographics and Baseline Characteristics of Patients

\begin{tabular}{|c|c|}
\hline & $\begin{array}{l}\text { All Patients } \\
\quad \mathrm{N}=200\end{array}$ \\
\hline \multicolumn{2}{|l|}{ Sex (n [\%]) } \\
\hline Female & 109 [54.5] \\
\hline Male & 91 [45.5] \\
\hline \multicolumn{2}{|l|}{ Patient Primary Complaint (n reporting [\%] for each) } \\
\hline Cough, fevers, or night sweats & $52[26.0]$ \\
\hline Diarrhea, vomiting or abdominal pains & $42[21.0]$ \\
\hline Headache, seizures or altered mental status & $37[18.5]$ \\
\hline Weakness or palpitations & $21[10.5]$ \\
\hline Difficulty breathing and other respiratory complaints & $8[4.0]$ \\
\hline Renal dysfunction & $8[4.0]$ \\
\hline Other Conditions ${ }^{\dagger}$ & $32[16.0]$ \\
\hline Duration of hospital stay (median [ IQR] days) & $7[5-10]$ \\
\hline Patient age (mean, range, SD years) & $37.5[30.8-45.2]$ \\
\hline \multicolumn{2}{|l|}{ ART status on admission ( $\mathrm{n}[\%]$ ) } \\
\hline Previously on ART & $184[92.0]$ \\
\hline Duration on ART $>12$ months & $107[58.1]$ \\
\hline
\end{tabular}

$\underline{\text { Inpatient Advanced HIV Diagnostic and Treatment Cascades }}$

Of the 200 patients in this study, 92\% were currently on ART at time of admission, including 58\% for more than 12 months (107/184) (Figure 2A). Clinicians requested HIV VL for 146 patients yet only $26(18 \%)$ had results available at the end of inpatient care. VLs were requested on average at day 1.5 of admission and the mean turnaround time was 6.5 days when reagents were available else not at all. When results were available, over half ( 14 of $26,54 \%$ ) had HIV VL >1,000copies/ml (median 5.5 Log [IQR 5.3 - 6.1Log]; suggesting ART failure or disengagement from care. Among the 184 ART-experienced patients, 33 (18\%) were switched during hospitalization to either second-line therapy or for reasons of renal or hepatic dysfunction. 
Figure $2 \mathrm{~B}$ shows monitoring of CD4 counts and appropriate use of CTX at discharge. Among participants reviewed, a clinician requested CD4 count for 87\%; however, results were documented in only $44 \%$ (77 of 174 ), on average on the $5^{\text {th }}$ day of admission. When resulted, CD4 counts were $<350$ cells $/ \mathrm{mm}^{3}$, the threshold at which CTX is indicated for Ol prophylaxis, $77 \%$ of the time (59 of 77). There was evidence of CTX prescription (on admission, during admission, or at discharge) for few participants (18 of 200 participants), and only 31\% of those with CD4 count $<350$ cells $/ \mathrm{mm}^{3}$ (18 of the 59 ).

Among participants, 28 were on ATT on admission, (Figure 2C). Of the remaining 172, 107 (62\%) were assessed for TB. Assessment included screening for typical TB symptoms in 43 (40\%) i.e. cough, fever, weight loss or night sweats. TB test was requested at an average of 3.3 days of admission. For Xpert MTB/RIF, time to results was 4.4 days from admission. However, only 60 patients had a documented test result, where 46 were negative on Xpert MTB/RIF or LAM. By discharge or death, 36 patients had newly initiated ATT, including 4 with a positive result and an additional 32 in whom there was high clinical suspicion. Nine of the 14 patients (64\%) with a positive result had died before the results were available.

WHO criteria for CrAg testing was met by $24 \%$ of patients ( 47 of the 200 patients), i.e. 18 with symptoms suggestive of meningitis and an additional 29 presented with CD4 counts less than 100 cells $/ \mathrm{mm}^{3}$ (Figure 2D) (9). Serum CrAg testing was not routinely available, and 38 of the 47 eligible (81\%) accepted to have their CSF tested. Six of the seven patients with a positive $\mathrm{CrAg}$ were discharged on fluconazole, one had died whilst receiving fluconazole, and two additional patients received fluconazole prophylactically. 
Across all diagnostic tests, we also assessed the time from test resulting in LIS to filing in the chart. Over $95 \%$ of results in the computer and file were concordant. The time from resulting in the LIS system and filing was $<1$ day on average (median 3 days [IQR 1-6 days].

\section{Discussion}

We conducted a retrospective, descriptive study of the in management for patients with advanced HIV disease compared to guideline recommendations to identify significant gaps in advanced HIV care at a large volume inpatient tertiary setting in Zambia where HIV burden is high. Although they are widely diagnosed in these settings, Ols are likely under-ascertained based on our data, in part due to incomplete recognition of the syndrome of advanced HIV, as well as challenges in laboratory diagnosis(18-20). Additionally, implementation of guidelines takes time, and this could have been another contributing factor to low guideline adherence. These data also suggest that inpatient settings offer an opportunity to identify ART treatment failure early enough.

Traditionally, CD4 count has been used as a gateway test to LAM and CrAg in the WHO guidelines (i.e., LAM and CrAg should only be performed following CD4 results) (9); however, the timing of LAM and CrAg tests when CD4 count is unknown should be established. In settings with high advanced HIV burden, LAM and CrAg could be done on all admitted PLHIV, even before CD4 count results are available, to shorten the time lapse of rapid tests that can be performed at bedside, avoiding further delay in diagnostics, especially if lab reagent supply constraints limit CD4 testing. 
Many severely ill patients cannot produce sputum for TB testing $(18,20)$; therefore, we considered either sputum Xpert MTB/RIF or LAM as evidence of TB testing. Even so, one-third of patients had neither test, due to the absence of a clinician request or for lack of results. Urine TB-LAM testing was introduced in the Zambia consolidated treatment guidelines in 2017 but implementation was delayed to late 2020. Previously published research at UTH revealed the high burden of TB and the positive impact of TB-LAM testing(20); however, the expected reagent supply to meet the demand has not materialized. In a multi-country meta-analysis, Barr et al. found that diagnostic delays and delayed initiation of ATT increase the risk of TB-related deaths in PLHIV, and that bedside LAM guided initiation of ATT is associated with reduced inpatient mortality (18). In our study, time lag to receiving results was observed in 9 of 14 patients (64\%) with newly diagnosed TB who died before they could receive their results - akin to an autopsy study that found $26 \%$ of patients with respiratory symptoms had undiagnosed TB at time of death (21). The 2015 National TB Prevalence study entailed household sampling of people in various communities across the country, conducting questionnaire-based symptom screening and sputum examination, plus chest $x$-ray where necessary. They found that $49 \%$ of sputum positive patients in the community had previously sought care at a public facility and concluded that health care staff lacked the knowledge and index of suspicion to diagnose TB in time (22). Our data shows instead that health care workers ordered appropriate diagnostics in $86 \%$ of the patients but a large proportion of these tests were not conducted due to logistical challenges and other impediments.

Cryptococcal meningitis remains a major cause of HIV-associated death $(19,23,24)$. We found low uptake of CrAg testing outside of cerebral spinal fluid (CSF) testing in patients with 
suspected meningitis. Serum CrAg testing was not offered to any of the patients, and acceptance of lumbar puncture (LP) may have been affected by societal norms (25).We, however, did not assess the proportion of patients who declined LP. Although recommended, pre-emptive treatment with fluconazole in patients who are serum CrAg positive but without overt meningitis was not widely implemented.

In addition to describing gaps in screening and management of Ols, we also found that hospitalization presents a potential opportunity for ART monitoring. Demand for VL by clinicians was high but only $\sim 15 \%$ ultimately received results; this was similar to a Malawian study of patients admitted with stage 3 or 4 HIV disease and on ART for more than one year, whereby $77 \%$ qualified for VL testing yet only $14 \%$ had results eight weeks post discharge (26). We propose that reflex viral load testing on admission for PLHIV may improve quality of care, as often the outpatient files may not be available to the clinicians at time of admission. Challenges of viral load testing in LMIC are well established with cost of reagents and consumables ranked highly (27). Even in higher middle-income countries, with well-funded HIV programs, clinicians often fail to routinely utilize viral load results available to optimize patient regimens (28). In our patient population, results revealed that $50 \%$ were non-suppressed, which compares to $10-15 \%$ nonsuppression nationally (29). This suggests that universal VL testing would likely identify a larger number of non-suppressed patients, which would then prompt early intervention by clinicians. As the majority of patients had advanced HIV as evidenced by low CD4 counts, strengthened adherence or regimen switches could be lifesaving in the near future.

The limitations of this study are largely attributable to the retrospective design, which has a greater propensity for missing data, the lack of qualitative explanatory data to shed light on the 
reasons for gaps in cascades and the late roll out of the WHO adapted Advanced HIV Disease guidelines. Selection bias may have been introduced due to the missing files from the patients who left with their files for review at their primary health centers as opposed to the UTH. Because all data are missing for these clients (not just the specific variables we are interested in) we are not able to conduct a sensitivity analysis to compare the 'missing' population to the 'available' population. Additionally, the facility presently does not have an electronic health record system which would contribute to availability of patient files and reporting of lab results. Whilst nearly all PLHIV were ART-experienced, it is difficult to conclude what proportion of these clients required additional VL testing without access to the outpatient care file.

Regardless, as the majority of patients with advanced HIV disease present at inpatient settings, this is an appropriate population to assess for lapses in patient care. We believe that the charts that were available are representative of the patient population we are trying to describe as the sickest patients with advanced HIV disease are often those who are retained in care at this tertiary center.

Future research is recommended to assess statistical relationships between services provided and outcomes with robust considerations of adequate power and sampling methods. Strengths include that the study was done at the largest tertiary center in the country with the largest resource allocation and the use of the LIS, which allowed us to accurately measure test resulting in the hospital.

\section{Conclusions}


Observed diagnostic delays and inadequate lab support remain important factors related to inpatient and post discharge mortality. In addition to attainment of $90-90-90$ treatment targets, treatment and prevention of Ols such as tuberculosis and Cryptococcal meningitis in PLHIV is an important marker of HIV program success and should be monitored as marker of quality. Based on the results of this descriptive study, we propose that reflex admission screening with HIV VL, urine LAM, and serum CrAg on for all PLHIV regardless of CD4 count result may significantly improve quality of HIV care and outcomes.

Word count: 3099 
Figure 1: Study flow Diagram

\section{Study Flow Diagram}

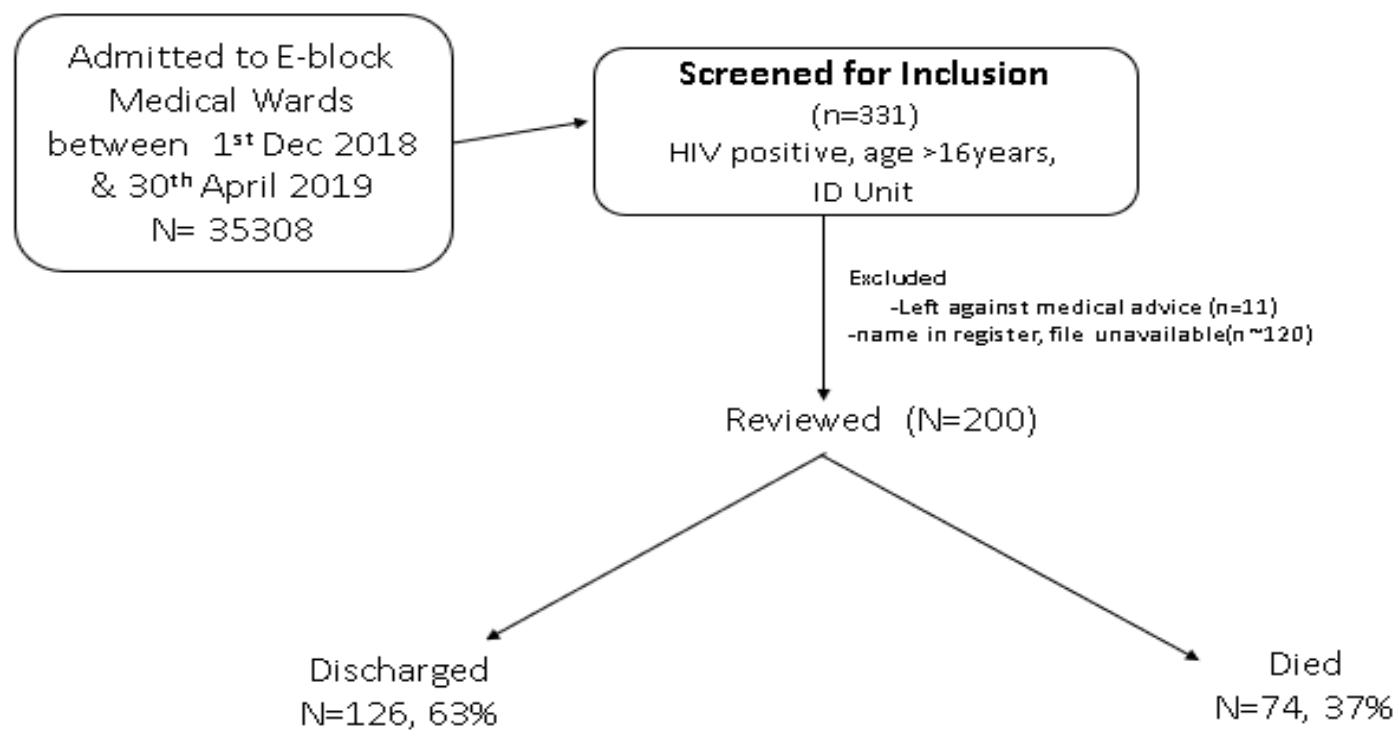


Figure 2: Advanced HIV disease testing cascades

A 250 Viral Load Monitoring

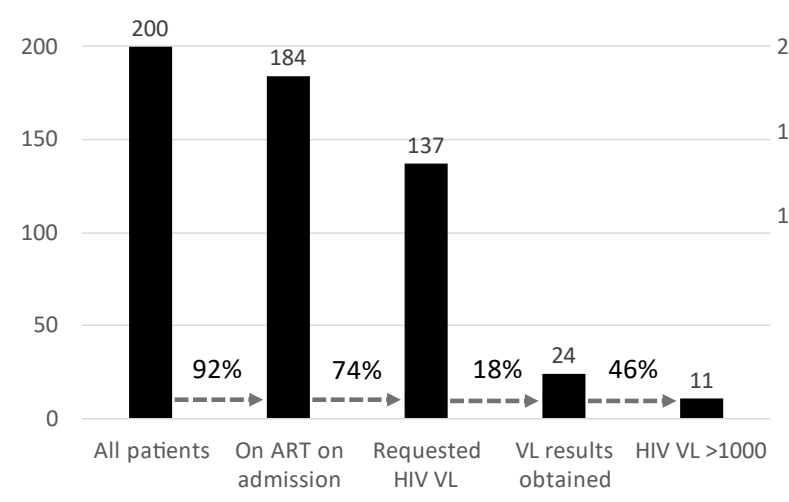

C 250

TB Diagnostic Testing

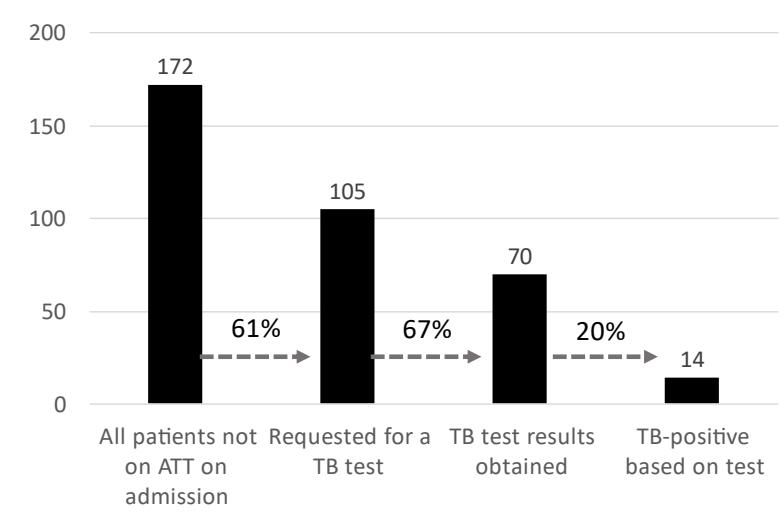

B

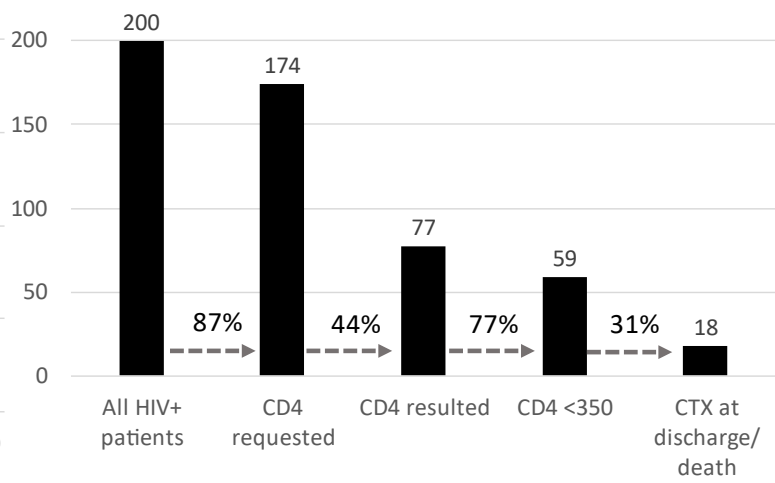

D 250

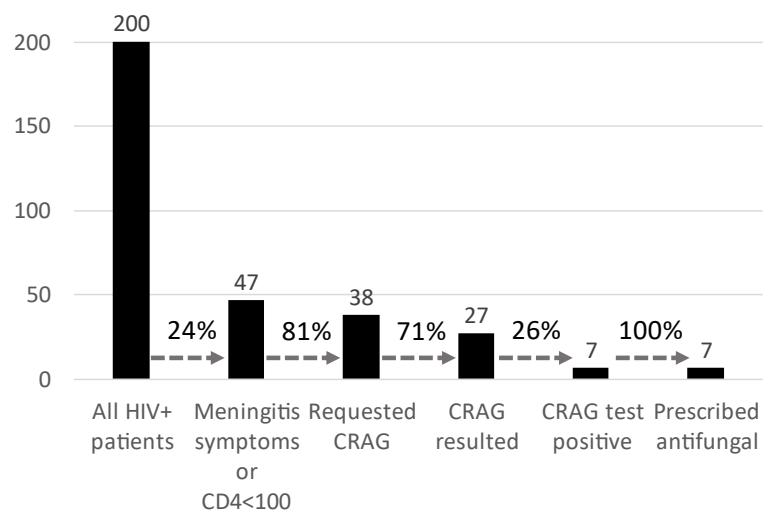

A. Number of patients receiving viral load monitoring. B. Number of patients receiving monitoring of CD4 count. C. Number of patients receiving TB Diagnostic testing. D. Number of patients undergoing cryptococcal diagnostics. 
1. UNAIDS. Global HIV \& AIDS Factsheet 2018- State of the epidemic. UNAIDS 2018. Available from https://www.unaids.org/en/resources/fact-sheet accessed 05 April 2021

2. Carmona S, Bor J, Nattey C, Maughan-Brown B, Maskew M, Fox MP, et al. Persistent High Burden of Advanced HIV Disease among Patients Seeking Care in South Africa's National HIV Program: Data from a Nationwide Laboratory Cohort. Clinical Infectious Diseases. 2018 Mar 4;66:S111-7.

3. Haachambwa L, Kandiwo N, Zulu PM, Rutagwera D, Geng E, Holmes CB, et al. Care Continuum and Postdischarge Outcomes among HIV-Infected Adults Admitted to the Hospital in Zambia. Open Forum Infectious Diseases. 2019;6(10):1-6.

4. Jahagirdar D, Walters MK, Novotney A, Brewer ED, Frank TD, Carter A, et al. Global, regional, and national sex-specific burden and control of the HIV epidemic, 1990-2019, for 204 countries and territories: the Global Burden of Diseases Study 2019. The Lancet HIV. 2021 Oct;8(10):e633-51.

5. Kerkhoff AD, Sikombe K, Eshun-Wilson I, Sikazwe I, Glidden D v., Pry JM, et al. Mortality estimates by age and sex among persons living with HIV after ART initiation in Zambia using electronic medical records supplemented with tracing a sample of lost patients: A cohort study. PLoS Medicine. 2020 May 1;17(5).

6. Dawood H, Hassan-Moosa R, Zuma NY, Naidoo K. Mortality and treatment response amongst HIV-infected patients 50 years and older accessing antiretroviral services in South Africa. BMC Infectious Diseases. 2018 Apr 10;18(1).

7. Workie KL, Birhan TY, Angaw DA. Predictors of mortality rate among adult HIVpositive patients on antiretroviral therapy in Metema Hospital, Northwest Ethiopia: a retrospective follow-up study. AIDS Research and Therapy. 2021 Dec 1;18(1).

8. UNAIDS. Global Aids Report 2020- Seizing the moment (Executive Summary) [Internet]. Seizing the Square. Geneva; 2020. Available from:

https://www.unaids.org/sites/default/files/media_asset/2020_global-aids-report_executivesummary_en.pdf

9. World Health Organization. Guidelines for managing advanced HIV disease and rapid initiation of antiretroviral therapy. Licence: CC BY-NC-SA 3.0 IGO. 2017. 1-56.

10. Lebelonyane R, Mills LA, Mogorosi C, Ussery F, Marukutira T, Theu J, et al. Advanced HIV disease in the Botswana combination prevention project: prevalence, risk factors, and outcomes. AIDS (London, England). 2020;34(15):2223-30.

11. Ministry of Health Government of the Republic of Zambia. Guidelines for Treatment and Prevention of HIV Infection. 2020.

12. Mfinanga S, Chanda D, Kivuyo SL, Guinness L, Bottomley C, Simms V, et al. Cryptococcal meningitis screening and community-based early adherence support in people with advanced HIV infection starting antiretroviral therapy in Tanzania and Zambia: An open-label, randomised controlled trial. The Lancet. 2015;385(9983):217382.

13. Manosuthi W, Wiboonchutikul S, Sungkanuparph S. Integrated therapy for HIV and tuberculosis. AIDS Research and Therapy. 2016;13(1):1-12.

14. Hakim J, Musiime V, Szubert AJ, Mallewa J, Siika A, Agutu C, et al. Enhanced Prophylaxis plus Antiretroviral Therapy for Advanced HIV Infection in Africa. New England Journal of Medicine. 2017;377(3):233-45. 
15. Cohen MS, Chen YQ, McCauley M, Gamble T, Hosseinipour MC, Kumarasamy N, et al. Antiretroviral Therapy for the Prevention of HIV-1 Transmission. New England Journal of Medicine. 2016;375(9):830-9.

16. World Health Organisation. MANAGING ADVANCED HIV DISEASE AND RAPID INITIATION OF ANTIRETROVIRAL THERAPY. 2017.

17. Ministry of Health Government of the Republic of Zambia. Zambia Consolidated Guidelines for Treatment and Prevention of HIV Infection. 2016.

18. Barr DA, Lewis JM, Feasey N, Schutz C, Kerkhoff AD, Jacob ST, et al. Mycobacterium tuberculosis bloodstream infection prevalence, diagnosis, and mortality risk in seriously ill adults with HIV: a systematic review and meta-analysis of individual patient data. The Lancet Infectious Diseases. 2020;1-11.

19. Molloy SF, Kanyama C, Heyderman RS, Loyse A, Kouanfack C, Chanda D, et al. Antifungal combinations for treatment of cryptococcal meningitis in Africa. New England Journal of Medicine. 2018;378(11):1004-17.

20. Andrews B, Semler MW, Muchemwa L, Kelly P, Lakhi S, Heimburger DC, et al. Effect of an early resuscitation protocol on in-hospital mortality among adults with sepsis and hypotension: A randomized clinical trial. JAMA - Journal of the American Medical Association. 2017;318(13):1233-40.

21. Bates M, Mudenda V, Shibemba A, Kaluwaji J, Tembo J, Kabwe M, et al. Burden of tuberculosis at postmortem in inpatients at a tertiary referral centre in sub-Saharan Africa: A prospective descriptive autopsy study. The Lancet Infectious Diseases. 2015;15(5):54451 .

22. Kapata N, Chanda-Kapata P, Ngosa W, Metitiri M, Klinkenberg E, Kalisvaart N, et al. The prevalence of tuberculosis in Zambia: Results from the first national TB prevalence survey, 2013-2014. PLoS ONE. 2016;11(1):2013-4.

23. Siddiqi OK, Ghebremichael M, Dang X, Atadzhanov M, Kaonga P, Khoury MN, et al. Molecular Diagnosis of Central Nervous System Opportunistic Infections in HIV-Infected Zambian Adults. Clinical Infectious Diseases. 2014;58:1771-7.

24. Mwaba P, Mwansa J, Chintu C, Pobee J, Scarborough M, Portsmouth S, et al. Clinical presentation, natural history, and cumulative death rates of 230 adults with primary cryptococcal meningitis in Zambian AIDS patients treated under local conditions. Postgraduate Medical Journal. 2001;77(914):769-73.

25. Thakur K, Mateyo K, Hachaambwa L, Kayamba V, Mallewa M, Mallewa J, et al. Lumbar puncture refusal in Sub-Saharan Africa: a call for further understanding. Vol. 84, American Academy of Neurology. 2015.

26. Craik A, Patel P, Patel P, Mallewa J, Malisita K, Bitilinyu-Bangoh J, et al. Challenges with targeted viral load testing for medical inpatients at Queen Elizabeth Central Hospital in Blantyre, Malawi. Malawi Medical Journal. 2016;28(4):179-81.

27. Roberts T, Cohn J, Bonner K, Hargreaves S. Scale-up of Routine Viral Load Testing in Resource-Poor Settings: Current and Future Implementation Challenges. 2016;62:1043-8.

28. Raizes E, Hader S, Birx D. The US President' s Emergency Plan for AIDS Relief ( PEPFAR ) and HIV Drug Resistance : Mitigating Risk, Monitoring Impact. 2018;216(January):2017-9.

29. Global Aids Monitoring 2019. Country progress report - Zambia. 2019; 


\section{$\underline{\text { Declarations }}$}

\section{Ethics approval and consent to participate}

The study was approved by UTH, the University of Zambia Biomedical Research Ethics Committee (REF. NO. 012-02-19), the University of Maryland Baltimore institutional review board (IRB) (FWA\#00007145), and the National Health Research Authority. A waiver of informed consent was obtained from the IRBs and no patient identifiers were used.

\section{Consent for publication}

Not applicable

\section{Availability of data and materials}

All datasets generated or analysed in this current study are available from the corresponding author on reasonable request.

\section{Competing interests}

The authors declare that they have no competing interests.

\section{Funding}

This study was conducted by Zambian physicians working at UTH, in conjunction with American physicians who volunteer their time at UTH. This study was conducted to better understand gaps in care for PLHIV at UTH to improve their care. We have no dedicated funding for this study. Therefore, we are asking for a waiver or at least a discount of the article processing charge. Thank you for your consideration of this request

6. Authors' contributions

MV, MY, LM, SF, SL, LH, KS, and CC prepared the study design, and initial ethical approvals; MY, MM and NM completed the data collection; and NM, VN, SS, KS, BL, $\mathrm{LM}$ and $\mathrm{CC}$ were major contributors in preparation of the final manuscript. All authors read and approved the final manuscript.

\section{Acknowledgements}

The authors gratefully acknowledge the patients and health care workers who made this work possible, as well as the support from University Teaching Hospital.

\section{Authors' information (optional)}

Not applicable 